\title{
NEURODEGENERATION AS AN EARLY SIGN OF DIABETIC RETINOPATHY
}

\author{
Nadia Artha Dewi ${ }^{1}$, Muhammad Arfan ${ }^{1}$, Herisa Rahmasari ${ }^{1}$, Mutiara Kristiani Putri ${ }^{1}$, Rulli Rosandi ${ }^{2}$, Shahdevi Nandar \\ Kurniawan $^{3}$ \\ Correspondence: nadia_dewi@ub.ac.id \\ ${ }^{1}$ Department of Ophthalmology, Medical Faculty Universitas Brawijaya, Saiful Anwar General Hospital \\ ${ }^{2}$ Department of Internal Medicine, Medical Faculty Universitas Brawijaya, Saiful Anwar General Hospital \\ ${ }^{3}$ Department of Neurology, Medical Faculty Universitas Brawijaya, Saiful Anwar General Hospital
}

\section{Article History:}

Received: December 12, 2021

Accepted: December 31, 2021

Published: January 1, 2022

\section{Cite this as:}

Dewi NA, Arfan M, Rahmasari $H$, Putri MK, Rosandi R, Kurniawan SN. Malang Neurology Journal; 2022.8:58-63. DOI:

http://dx.doi.org/10.21776/ub.mnj .2022 .008 .01 .12

\begin{abstract}
Diabetic retinopathy is major cause of visual impairment and blindness in diabetic patients worldwide. The concept of diabetic retinopathy as vascular disease has established into not only microvascular complication but also neurodegeneration problems. Neurodegeneration plays an important role in pathogenesis of diabetic retinopathy. In fact, neuroretinal changes in diabetes can take place even before vasculopathy can be clinically detected. This condition is marked by accelerated loss of neurons due to apoptosis, particularly in the inner retinal layer. The characteristic of neurodegeneration can be detected through retinal imaging and electrodiagnostics. This review is very crucial, because identifying the pathophysiology of diabetic neurodegeneration better, we may be able to provide interventions using the appropriate therapy. We may also be able to utilize these diagnostic tools for early detections of diabetic retinopathy, thus preventing blindness due to diabetes.
\end{abstract}

Keywords: Neurodegeneration, diabetic retinopathy, neurovascular unit, retinal nerve fiber layer, review

\section{Introduction}

Diabetes mellitus is the most serious metabolic disorder, characterized by hyperglycemia along with disorders of lipid metabolism, carbohydrate, and protein. Chronic diabetes leads to various complications, such as retinopathy, neuropathy, and nephropathy. Diabetic retinopathy is major complication of diabetes mellitus. The prevalence of diabetic retinopathy in Indonesian adults with type 2 diabetes mellitus is $43.1 \%$ with the prevalence of vision threatening diabetic retinopathy (VTDR) is $26.3 \% .^{1}$

So far, diabetic retinopathy has been considered a disease resulting from the primary microvascular complications of diabetes. In establishing a diagnosis, its effects must also be observed on the retinal vasculatures, for instance, microaneurysm, macular edema, and neovascularization. The therapy options currently in use comprise laser photocoagulation and intravitreal anti-VEGF injection. However, these therapies can only be undertaken once the disease has reached its late-stage and the patient is already experiencing a reduced vision. ${ }^{2,3}$

The advancements of our understanding of the cellular changes taking the retina of patients with diabetes, combined with improvements in retinal imaging, has given birth to a novel concept in which diabetic retinopathy can be perceived as a disease of the retina's neurovascular unit (NVU), which refers to the functional attachment and interdependency of neurons, glia, and vasculatures integrating for dictating normal retinal function. The conceptualization of diabetic retinopathy as a disease of the NVU expands our horizon in regard to the types of cells contributing to the development and progression of diabetic retinopathy. ${ }^{4-6}$

Recent research suggests that the neuroretinal changes taking place in a diabetic condition have shifted the paradigm that the vascularizations is the sole mechanism that explains the occurrence of retinopathy. In fact, neuroretinal changes in diabetes can take place even before vasculopathy can be clinically detected. This condition is marked by accelerated loss of neurons due to apoptosis, particularly in the inner retinal layer. As a consequence, these initial changes in the neuroretina may allow early detection of diabetic retinopathy. ${ }^{4-6}$

In this literature review, we will be discussing the newest developments of the involved mechanisms of neurodegeneration in diabetic retinopathy, early detection signs of neurodegeneration that consist of both histologic and structural changes.

\section{The Roles of Neurovascular Unit in Retina}

Just how the blood-brain barrier has a tight junction between the capillary endothelial cells, the retina also possesses a neurovascular unit (NVU) positioned near the retinal pigmented epithelium (RPE), which is on the outer blood-retina barrier (BRB). The function of NVU in the retina is to maintain the integrity of $\mathrm{BRB}$ by dynamically 
regulating blood flow according to the retina's metabolism. Specifically, NVUs in the retina have a capillary network between the Ganglion Cell Layer (GCL) and the Inner Nuclear Layer (INL). An NVU includes neural cells (ganglion, amacrine, horizontal, and bipolar cells), glial cells (Müller cells and astrocytes), microglia, and vascular cells (pericytes and endothelial cells).Müller cells are radial cells located in the entire retinal layer and comprise $90 \%$ of the total glial cell composition. Their roles are to maintain retinal function and metabolism by producing neurotrophic factors, recycle glutamate neurotransmitters, and control extracellular electrolyte balance. ${ }^{7-10}$

Astrocytes are only bound to the Retinal Nerve Fiber Layer (RNFL) and there is only so much present in the ganglion layer. Their functions are to produce neurotrophic factors, provide mechanical support for neurons, and undertake maintenance of BRB. This type of glial cell is microglia, which is a tissue macrophage of high importance for retinal homeostasis and disease recovery and progressivity. Endothelial cells included in the inner BRB and pericytes are vascular units that form the tight junction in the retinal blood vessels. ${ }^{7-10}$

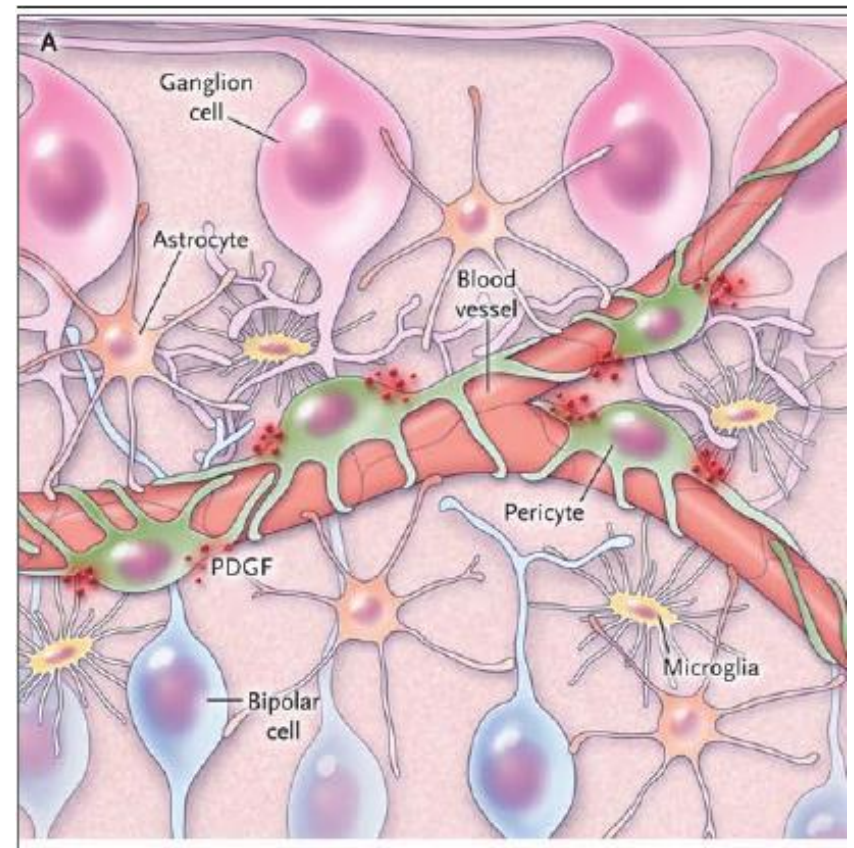

Figure 1. Neurovascular Unit. ${ }^{8}$ Neurovascular unit shows complex structure and interdependency between neural cells (astrocyte, glial cells), and vascular cells (pericyte) as inner blood retinal barrier.

\section{The Pathophysiology of Diabetic Retinal Neurodegeneration}

Neurodegeneration contributes to the pathophysiology of diabetic retinopathy, and its process begins since the early onset of diabetes in patients. Diabetes mellitus induces the apoptosis of retinal ganglion cells, amacrine cells, and Müller cells. Neurodegeneration in diabetic retinopathy is the effect of decreased signals of insulin receptors and systemic hyperglycemia that have a direct implication to how intensive metabolic control has an effect on the development of retinopathy.In a diabetic condition, the equilibrium of NVU will be disrupted. The processes of innate immunity, complement system, and activated microglia will take place and result in early damage to the NVU. These are the factors of how Diabetic Retinal Neurodegeneration (DRN) occurs. ${ }^{11}$

Disturbances of the neurosensory retina in diabetes can take place through various mechanisms, which are divided into inflammatory, metabolic, genetic/epigenetic. Hyperglycemic condition alters the biochemical pathway (elevation of polyol pathway, hexosamine pathway, protein kinase C [PKC], and advanced glycation end-products [AGEs]), which will induce the apoptosis of ganglion, amacrine, and Müller cells. The expression of the glial fibrillary acidic protein (GFAP) in Müller cells and microglial reaction will increase, thus causing chronic glutamate toxicity, resulting in an imbalance in the synthesis of neuroprotective factors in the retina, and increasing oxidative stress and inflammation. These are the primary factors in the process of ocular neurodegeneration. $8,11,12$

The first noticeable change in diabetes is the decline of astrocyte cell count and changes in protein expression with the ensuing inner retina hypoxia and functional deficit in ganglion cell response. The Müller cell dysfunction due to chronic hyperglycemia leads to the release of numerous growth factors and cytokines. The reactivity of Müller cells is suspected as a neuroprotective factor, albeit contributing to the occurrence of neurodegeneration. This is mainly due to the decrease in Müller cells' ability, such as the malfunction of glutamate uptake, expression of nucleoside triphosphate diphosphohydrolase 1 (NTPDase1), allow the formation of extracellular adenosine. ${ }^{11}$

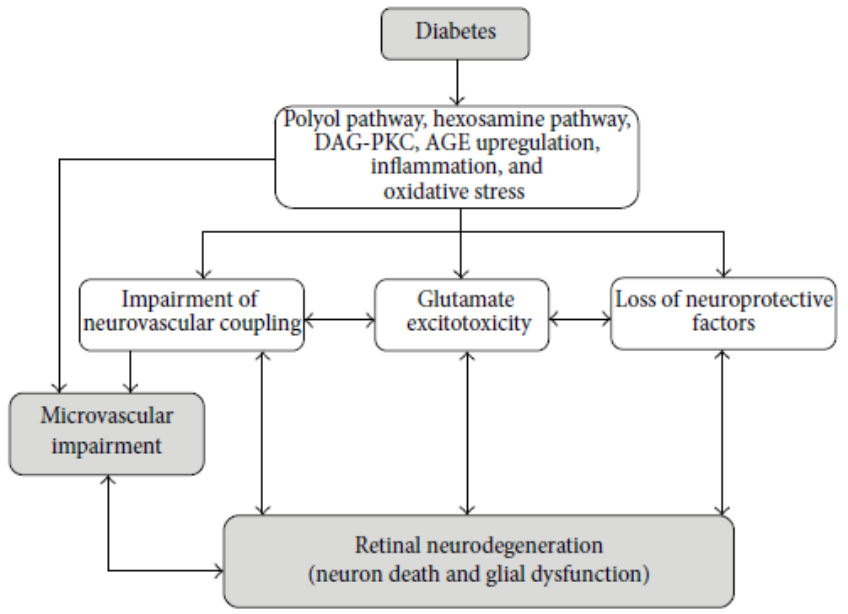

Figure 2. Pathophysiology of Diabetic retinal neurodegeneration. ${ }^{8}$ The biochemical changes caused by diabetes affect neuronal death and glial dysfuction through glutamate excitotoxicity, loss of neuroprotective factors, and impairment of neurovascular coupling.

\section{Glutamate Excitotoxicity}

The primary neurotoxic metabolite related to retinal neurodegeneration is glutamate. Glutamate is a major primary excitatory neurotransmitter in the retina and is included in the neurotransmitter the photoreceptors to bipolar cells that will be transmitted to ganglion cells. The underlying condition leading to the accumulation of glutamate in diabetes is the increased production of glial cells, which depletes Müller cells specific for glutamate synthesis, lessens the retina's ability to oxidize glutamate into $\alpha$-ketoglutarate, and dysfunction of glutamate uptake 
by glial cells. An increase in extracellular glutamate concentration and on synapse triggers excitotoxicity where a surplus of glutamate stimulation will result in excessive intracellular calcium response in postsynaptic neurons, ultimately leading to apoptosis. ${ }^{8,12}$

\section{Loss of Neuroprotective Factors}

The synthesis of retinal neuroprotective that combats neurotoxic effects that cause retinal neurodegeneration. The loss of neuroprotective factors or the reduction of their effectiveness plays a role in retinal neurodegeneration, among which are the pigment epithelium-derived factor (PEDF), somatostatin (SST), and erythropoietin that hold a vital role. PEDF is a neuroprotective factor and antiangiogenic factor that is downregulated in diabetic retinopathy. PEDF protects neurons from glutamatemediated neurodegeneration, and latest research reports that PEDF increases the expression of glutamine synthetase enzyme in early diabetic retinopathy phase. SST is a neuromodulator and angiostatic factor in the retina and is mainly synthesized in the retinal pigment epithelium (RPE). The level of SST produced by the retina can decrease with the high presence of it in the vitreous of those with diabetes. $6,8,12$

Erythropoietin is a physiological stimulus potential for the mobilization of endothelial progenitor cells, playing a key role in the regulation of progenitor cells to the damaged retina. The retina is a tissue that is often and directly exposed to light sources, inducing photooxidation on lipids, especially polyunsaturated fatty acids (PUFA) most often found on the outer photoreceptor layer. The oxidized lipid is toxic to the retina and causes oxidative stress, leading to neurodegeneration. Neuroinflammation is a condition in which dysregulation of pro inflammatory factors involving microglia, monocyte and Muller cells occur. Chronic neuropathy affect the morphology of retinal ganglion cells, changes of the synaptic action, neurotransmitter, and cell loss. ${ }^{8,12,13}$

\section{Oxidative Stress}

Oxidative stress is also involved in a diabetic condition, partly because increased body weight correlates with insulin resistance. Diabetes-related hyperglycemia induces increased ROS production, which activates several adverse alternative pathways. This includes the formation of advanced glycation end products and the downregulation of soluble receptors, the activation of polyol aldose reductase activity with kinase $\mathrm{C}$ accumulation, the activation of poly (ADP-ribose) polymerase and cyclooxygenase 2, significant endothelial dysfunction, peroxynitrite and protein nitration, and changes in $\mathrm{Na}^{+} / \mathrm{K}^{+}$-ATPase pump function. All of these changes contribute heavily to neuronal, mitochondrial, and endothelial dysfunction in diabetes. ${ }^{10}$ One significant complication of diabetes greatly responsible for oxidative stress is diabetic neuropathy. This complication has been extensively studied, and the majority acknowledge that early and intensive glycemic control (that limits hyperglycemia-induced oxidative stress) is effective and crucial for the treatment/prevention of this condition. It has also been reported that disrupted neural blood flow may be involved in the development of this complication. Therefore, vascular dysfunction as a result of endothelial activation may also be involved. Changes in lifestyle such as diet and physical exercise are also proven beneficial for people with neuropathy diabetes. ${ }^{12,14,15}$

Neurodegeneration in diabetic retinopathy is an effect of the declining signal of insulin receptor and systemic hyperglycemia, which has a direct implication on how intensive metabolic control has an effect on the development of retinopathy. Latest research reveals that the depletion and degeneration of the retinal neural layer, particularly on the nerve fiber layer (NFL), begin early before the occurrence of vascular lesions. ${ }^{7,8}$

\section{Recent Findings in The Pathophysiology of Diabetic Retinal Neurodegeneration}

Galectin-3 is involved in the inflammation process, oxidative stress, and apoptosis. Serine racemase (SRR) and D-serine as its product contributes to neurotoxicity. Stress response protein regulated in development and DNA damage-response 1 (REDD1), can trigger apoptosis. The overexpression of REDD1 in a hyperglycemic condition will result in cell death. Several neuroprotective factors are found to decrease in DRN: $\alpha \mathrm{A}$-crystallin(molecular chaperone, regulating neuronal cell survival in multiple neurodegenerative conditions), STRT6 ((a NAD-dependent sirtuin deacylase, which modulates aging, energy metabolism, and neurodegeneration). Sigma-1 receptor (Sig1R) is a pluripotent modulator that has a number of functions related to retinal diseases, such as calcium regulation, oxidative stress, ion channel regulation, and molecular chaperone activity. This functions as a neuroprotector for ganglion cells and photoreceptor cells. ${ }^{11}$

\section{Evidence of Diabetic Retinal Neurodegeneration Prior to Clinical Signs of Retinopathy}

A prominent feature often found in DRN is the loss of neuron due to apoptosis. Histologically, this is evident in a number of studies, such as that of Yun et al., which demonstrated the underlying mechanism of diabetic retinopathy through angiopoietin-2 that induces astrocyte apoptosis through integrin avb5 communication. An in vivo study found that astrocyte loss and vascular leakage are inhibited by the injection of Ang2-neutralizing antibody. Another in vivo finding also revealed that the injection of anti $\alpha v \beta 5$-integrin antibody inhibits astrocyte loss in early diabetic retinopathy. ${ }^{16,17}$

An experimental study by Valverde et al. conducted on March 2011 and January 2012 suggests cell damage and apoptosis in human diabetic retinopathy related with increased pro-apoptosis molecule expression, which results in the activation of death receptors and injury of the glial fibrillar acidic protein (GFAP) mitochondria, and proapoptosis molecules (FasL, active caspase-8, truncated Bid (t-Bid), Bim, and active caspase-3) increase significantly in the neuroretina of patients with diabetes in comparison that of control patients. ${ }^{18}$

Fu et al. in 2015 conducted a study to obtain a better understanding of a specific type of cell most affected by 
apoptosis in DM, which demonstrates a 53\% decrease in Müller cell density 10 months after DM induction in mice, which also coincides with a reduction in the thickness of the inner nuclear layer (INL), ganglion cell layer (GCL), and outer nuclear layer (OUL). In an observation made on photoreceptor cells experiencing early apoptosis 4 weeks after the induction of diabetes mellitus (DM) in an animal model, with a significant reduction in outer nuclear layer thickness in 24 weeks. The accumulation of data from latest research apparently suggests that photoreceptor cells may hold an essential role in the development of diabetic retinopathy. An increase in oxidative stress as a hyperglycemia-related metabolic disorder is involved in the development of the microvascular change of diabetic retinopathy. ${ }^{19}$

If neuronal apoptosis continuously occurs, this will lead to decreased density and volume of retinal neural tissue. Fortunately, this condition can be detected in humans through retinal imaging that has been developed today. Neurodegeneration in diabetes can be detected even before any clinical signs of diabetes in the retina take place. The retinal ganglion cells and amacrine cells are the first neurons detected to undergo apoptosis due to diabetes. On the other hand, photoreceptor cells also experience increased apoptosis. This structure is visible using the optical coherence tomography (OCT). It useful to measure neurodegeneration through retinal layer accurately and quantitative, non-invasive and high resolution. ${ }^{13,20}$

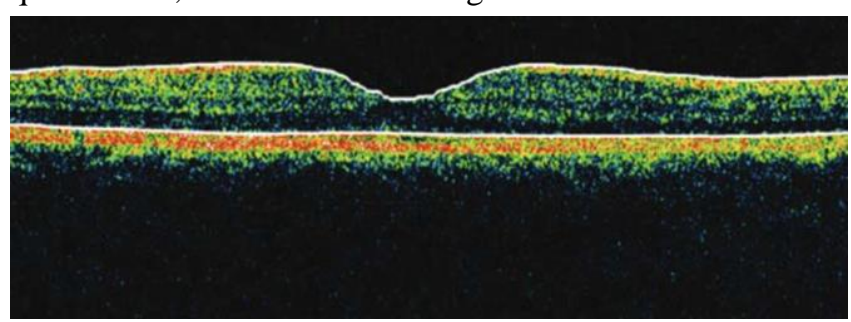

Figure 3. Retinal Layer in Normal Eye detected by OCT. We can see the retinal layers from inner to outer retina starts from Internal Limiting Membrane (ILM), Retinal Nerve Fiber Layer (RNFL),

Ganglion Cell Layer (GCL), Inner Plexiform layer (IPL), Inner Nuclear Layer (INL), Outer Plexiform layer (OPL), Outer Nuclear Layer (ONL), External Limiting Membrane (ELM),

Photoreceptors (PR), and Retinal Pigmented Epithelium (RPE).

An epidemiological study conducted by Gundongan et al., is a prospective case series throughout January 2012 and December 2014 exploring structural changes of the retina in type-1 diabetes without a clinical diagnosis of diabetic retinopathy (DR). Retinal nerve fiber layer (RNFL) thickness, ganglion complex cells (GCC) thickness, and macular thickness (MT) were measured in 90 patients with type-1 diabetes using the SD-OCT. The results were compared with 100 sex- and age-appropriate healthy controls. The results indicated that all RNFL, superior and inferior quadrant, and half of the superior from the peripapillary RNFL thickness are significantly thinner in patients with diabetes 19 compared with the control group $(\mathrm{p}<0.05)$. GCC thicknesses in the average macular, outer temporal superior and outer temporal inferior sectors were significantly thinner in diabetic patients $(\mathrm{p}<0.05) .{ }^{21}$

An epidemiological study undertaken by Scarinci et al. in 2016 as an evaluation of single retinal layer of patients with type-1 diabetes without or with early signs of diabetic retinopathy. The study involved 76 eyes from 38 patients with type-1 DM and 26 control eyes. Nine measurements of individual retinal layer thickness were obtained by using the spectral domain-optical coherence tomography (SDOCT) algorithm, which demonstrated a slightly thinner retinal neural fiber layer in all of the explored quadrants, with an insignificant reduction in eyes with type-1 DM compared with control eyes. ${ }^{24}$

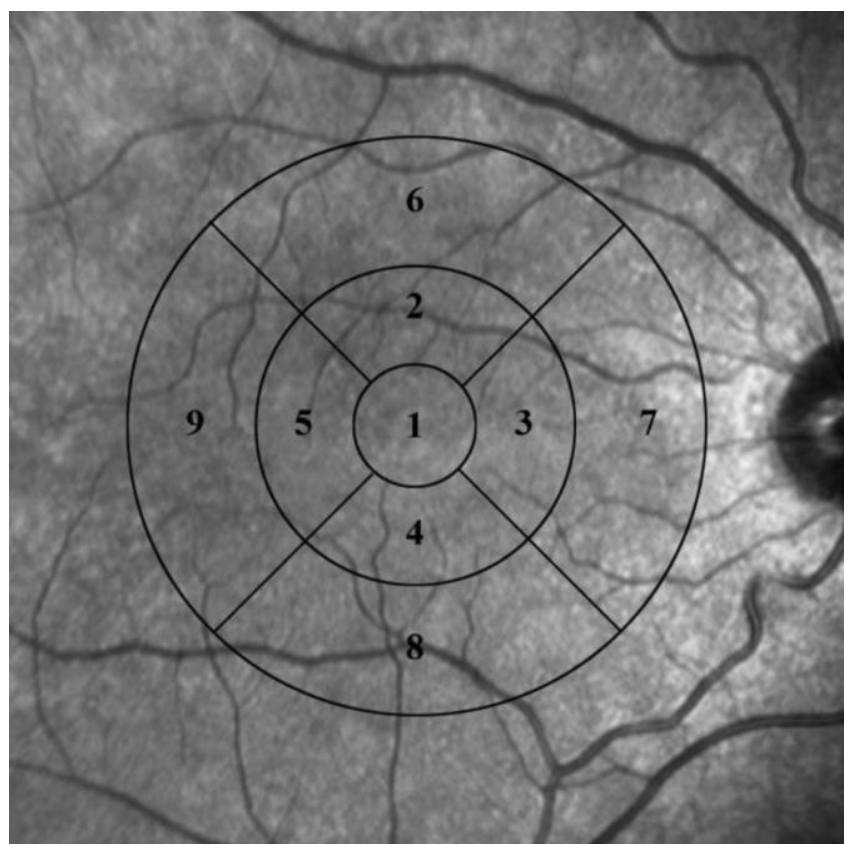

Figure 4. Macular thickness mapping using OCT. ${ }^{20}$ The zone were grouped in 9 sectors.

A cross-sectional study by Carpineto et al. from January 2013 to July 2014 examined the neuroretinal changes in patients with type-2 diabetes without diabetic retinopathy (DR) or non-proliferative diabetic retinopathy and signs of diabetic macular edema. The thickness of the ganglion cellinner plexiform layer (GC-IPL_ and the retinal nerve fiber layer (RNFL) were measured after SD-OCT automatic segmenting scanning. The average thickness of GC-IPL is $80.6 \pm 8.1 \mathrm{~m}$ in patients with diabetes and $85.3 \pm 9.9 \mathrm{~m}$ in healthy controls $(\mathrm{p}=0.001)$. Moreover, after evaluating two different diabetes groups, the thickness of GC-IPL was 80.7 $\pm 8.1 \mu \mathrm{m}$ and $79.7 \pm 8.8 \mu \mathrm{m}$ in the non-DR group and the mild NPDR group, respectively (each $\mathrm{p}=0.001$ and $\mathrm{p}=$ 0.022 compared with healthy controls). The average RNFL thickness was $86.1 \pm 10.1 \mathrm{~m}$ in patients with diabetes and $91.2 \pm 7.3 \mathrm{~m}$ in controls $(\mathrm{p}=0.003)$. RNFL thickness was $86.4 \pm 10,2 \mu \mathrm{m}$ in the non-DR group and $84.1 \pm 9.4 \mu \mathrm{m}$ in the mild NDPR group $(\mathrm{p}=0.007$ and $\mathrm{p}=0.017$ compared with healthy controls, respectively). This study displays a significantly decreasing GC-IPL and RNFL value in the non-DR group and the mild NPDR group, respectively, with the healthy control. Such findings confirm the occurrence of neuroretinal changes in the early stages of diabetes, preceding microvascular damage. ${ }^{25}$

Electrodiagnostics also could access visual fuction before and after retinopathy. Multifocal electroretinography (mfERG) is the gold standard for assessing retinal function damage, displaying that there is a slowing of P1 implicit time and decreasing trace in neurodegeneration. Pattern electroretinogram (pERG) also could access ganglion cell activity wiyh decrease of amplitude in diabetic patient before retinopathy. So too is the visual evoked potential 
(VEP), which is an electrophysiological assessment method for evaluating the visual pathway of retinal ganglion cells to the occipital cortex caused by visual stimuli. The main function of VEP is to measure the functional integrity of the visual pathway from the retina to the optic nerves, up to the visual cortex. Generally, VEP is effective in identifying any damage on the retinal ganglion cells in diabetes. VEP abnormality in diabetes mellitus is indicated by the P100 latency display. Early detection of diabetic retinopathy with the help of VEP can help prevent loss of visual function. ${ }^{26-29}$

\section{Neurodegeneration as A Biomarker of Neurodegenerative Diseases}

There are similarities in the morphological characters between the retina and brain-derived tissues, allowing the retina to be used as an access for examining the pathology of the central nervous system. Neuroimaging modalities, like MRI, are yet able to detect subclinical disorders of the brain due to their relatively larger resolution $(<100-500$ $\mu \mathrm{m})$. As a result, OCT may be used as an alternative for detective neurodegenerative processes, such as diabetic peripheral neurodegeneration. ${ }^{6,30,31}$

\section{Conclusion}

In diabetes, damage is already happened to the neurons in the retina, even before any clinical signs of diabetic retinopathy are present. By identifying the pathophysiology of diabetic neurodegeneration better, we may be able to provide interventions using the appropriate therapy according to the factors causing neurodegeneration and those related to the retinal neuron cells.

Retinal imaging technology and electrodiagnostic rapidly developing today can recognize the presence of neurodegeneration noninvasively. Hopefully, we may be able to utilize these diagnostic tools as a means for early detections of diabetic retinopathy, thus preventing blindness due to diabetes.

Further research is necessary to determine the causing pathway of diabetic retinal neurodegeneration and damage to neurovascular units, so that we may better enhance our understanding and also provide suitable and effective therapy before the disease has reached the late-stage.

\section{Conflict of Interest}

The Authors declare that there is no conflict of interest.

\section{Acknowledgement}

None.

\section{References}

1. Sasongko MB, Widyaputri F, Agni AN, Wardhana FS, Kotha S, Gupta P, et al. Prevalence of diabetic retinopathy and blindness in Indonesian adults with type 2 diabetes. American Journal of Ophthalmology; 2017. 181:79-87. DOI: 10.1016/j.ajo.2017.06.019
2. Wang W, Lo Amy CY. Diabetic retinopathy: Pathophysiology and treatment. International Journal of Molecular Science; 2018. 19,1816.

DOI: $10.3390 /$ ijms 19061816

3. Sam E Mansour, David J Browning, Keye Wong, Harry W Flynn Jr, Abdhish R Bhavsar. The evolving traetment of diabetic retinopathy. Clinical Ophthalmology; 2020. 14 653-678.

DOI: $10.2147 /$ OPTH.S236637

4. Duh EJ, Sun JK, Stitt AW. Diabetic retinopathy: current understanding, mechanisms, and treatment strategies. JCI Insight; 2017. Jul;2(14).

DOI: $10.1172 /$ jci.insight.93751

5. Lechner J, O'Leary OE, Stitt AW. The pathology associated with diabetic retinopathy. Vision research; 2017. Oct 1;139:7-14.

DOI: 10.1016/j.visres.2017.04.003

6. Simó R, Stitt AW, \& Gardner TW. Neurodegeneration in diabetic retinopathy: Does it really matter? Diabetologia; 2018. 61(9):1902-1912.

DOI: $10.1007 / \mathrm{s} 00125-018-4692-1$

7. Usui, Y. Elucidation of pathophysiology and novel treatment for diabetic macular edema derived from the concept of neurovascular unit. JMA Journal; 2020. 3(3):201-207. DOI: 10.31662/jmaj.2020-0022

8. Lynch SK, \& Abràmoff MD. Diabetic retinopathy is a neurodegenerative disorder. Vision Research; 2017. 139:101-107. DOI: 10.1016/j.visres.2017.03.003

9. Barber AJ. Diabetic retinopathy: Recent advances towards understanding neurodegeneration and vision loss. Scienc China Life Science; 2015. 58:541-549. DOI: $10.1007 / \mathrm{s} 11427-015-4856-\mathrm{X}$

10. Rashid K, Akhtar-Schaefer I, \& Langmann T. Microglia in retinal degeneration. Frontiers in immunology; 1975. DOI: 10.3389/fimmu.2019.01975

11. Pillar S, Molsselev E, Sokolovska J, Grzybowski A. Recent development in diabetic retinal neurodegeneration: A literature review. Journal of Diabetes Research; 2020.

DOI: $10.1155 / 2020 / 5728674$

12. Simó R, Hernández C, Neurodegeneration is an early event in diabetic retinopathy: Therapeutic implications. British Journal of Ophthalmology; 2012. 96:1285-1290. DOI: 10.1136/bjophthalmol-2012302005

13. Barber AJ, Baccouche B. Neurodegeneration in diabetic retinopathy: Potential for novel therapies. Vision research; 2017. Oct 1;139:82-92.

DOI: 10.1016/j.visres.2017.06.014

14. Sohn EH, Van Dijk HW, Jiao C, Kok PH, Jeong W, Demirkaya N, \& Abràmoff MD. Retinal neurodegeneration may precede microvascular changes characteristic of diabetic retinopathy in diabetes mellitus. Proceedings of the National Academy of Sciences; 2016. 113(19):E2655-E2664. DOI: $10.1073 /$ pnas. 1522014113

15. Elena Vecino, F David Rodriguez, Noelia Ruzafa, Xandra Pereiro, Sansar C Sharma. Glia-neuron interactions in the mammalian retina. Progress in Retinal and Eye Research; 2016. Volume 51, Pages 140. ISSN: 1350-9462.

DOI: 10.1016/j.preteyeres.2015.06.003. 
16. Yun et al. Angiopoietin 2 induces astrocyte apoptosis via av $\beta 5$-integrin signaling in diabetic retinophaty. Journal Cell Death and Disease; 2016. Vol. 7, Pages 1-11. DOI: $10.1038 /$ cddis.2015.347

17. Ramirez JM, Trivin A, et al. Immunohistochemical study of human retinal astroglia. Vision Research Journal; 1994. Vol.34. Page 1935-1946. DOI: 10.1016/0042-6989(94)90024-8

18. Valverde AM, Miranda S, García-Ramírez M, González-Rodriguez Á, Hernández C, \& Simó R. Proapoptotic and survival signaling in the neuroretina at early stages of diabetic retinopathy. Molecular Vision; 2013. 19:47. Avalaible from: https://pubmed.ncbi.nlm.nih.gov/23335850/

19. Fu S, Dong S, Zhu M, Sherry D, Wang C, You Z, \& Le YZ. Müller glia are a major cellular source of survival signals for retinal neurons in diabetes. Diabetes; 2013. 64(10), 3554-3563. DOI: $10.2337 / \mathrm{db} 15-0180$

20. Srinivasan S, Dehghani C, Pritchard N, Edwards K, Russell AW, Malik RA, \& Efron N. Optical coherence tomography predicts 4-year incident diabetic neuropathy. Ophthalmic and Physiological Optics; 2017. 37(4):451-459. DOI: 10.1111/opo.12391

21. Gundogan FC, Akay F, Uzun S, Yolcu U, Cagiltay E, Toyran S. Early neurodegeneration of the inner retinal layers in type 1 diabetes mellitus. Ophthalmologica; 2016. 235(3):125-32. DOI: 10.1159/000442826

22. Loduca AL, Zhang C, Zelkha R, \& Shahidi M. Thickness mapping of retinal layers by spectraldomain optical coherence tomography. American Journal Of Ophthalmology; 2010. 150(6):849-855. DOI: $10.1016 /$ j.ajo.2010.06.034

23. Ctori I, \& Huntjens B. Repeatability of foveal measurements using spectralis optical coherence tomography segmentation software. PloS One; 2015. 10(6). DOI: 10.1371/journal.pone.0129005

24. Scarinci Fabio, et al. Single retinal layer evaluation in patients with type 1 diabetes with no or early signs of diabetic retinopathy: The first hint of neurovascular crosstalk damage between neurons and capillaries? Ophthalmologica; 2017. 237(4):223-231.

DOI: $10.1159 / 000453551$
25. Carpineto P, Toto L, Aloia R, Ciciarelli V, Borrelli E, Vitacolonna E, et al. Neuroretinal alterations in the early stages of diabetic retinopathy in patients with type 2 diabetes mellitus. Eye; 2016. 30(5):673-9. Avalaible from:

https://www.nature.com/articles/eye201613

26. Zafar Sidra et al. Retinal neurodegeneration as an early manifestation of diabetic eye disease and potential neuroprotective therapies. Current Diabetes Reports; 2019. 19(4):17. DOI: 10.1007/s11892-0191134-5

27. Lakhani E, Wright $\mathrm{T}$, Abdolell $\mathrm{M}$, Westall C. Multifocal ERG defects associated with insufficient long-term glycemic control in adolescents with type 1 diabetes. Invest Ophthalmol Vision Science; 2010. 51(10):5297-303. DOI: 10.1167/iovs.10-5200

28. Heravian J, Ehyaei A, Shoeibi N, Azimi A, OstadiMoghaddam H, Yekta AA, et al. Pattern visual evoked potentials in patients with type II diabetes mellitus. J Ophthalmic Vision Res; 2012. 7(3):225-30. Avalaible form:

https://www.ncbi.nlm.nih.gov/pmc/articles/PMC3520 591/

29. Corduneanu Angela CV, Ciobanu Natalia, Groppa Stanislav. Evaluation of visual pathways using visual evoked potential in patients with diabetic retinopathy. Romanian Journal of Ophthalmology; 2019. 4:4. Avalaible from:

https://pubmed.ncbi.nlm.nih.gov/31915735/

30. Xiao-hong Wu, Jing-wen Fang, Yin-qiong Huang, Xue-feng Bai, Yong Zhuang, Xiao-yu Chen, Xia-hong Lin. Diagnostic value of optic disc retinal nerve fiber layer thickness for diabetic peripheral neuropathy. Journal of Zhejiang University-Science B (Biomedicine \& Biotechnology); 2020. 21(11):911920. DOI: 10.1631/jzus.B2000225

31. Hafner J, Zadrazil M, Grisold A, Ricken G, KrennM, Kitzmantl D, et al. Retinal and corneal neurodegeneration and its association to systemic signs of peripheral neuropathy in type 2 diabetes. American Journal of Ophthalmology; 2020. 209:197-205. DOI: 10.1016/j.ajo.2019.09.010. 